\title{
Bubble Pinch-Off in a Rotating Flow
}

\section{Bergmann, Raymond; Andersen, Anders Peter; van der Meer, Devaraj; Bohr, Tomas}

\section{Published in:}

Physical Review Letters

Link to article, DOI:

10.1103/PhysRevLett.102.204501

Publication date:

2009

Document Version

Publisher's PDF, also known as Version of record

Link back to DTU Orbit

Citation (APA):

Bergmann, R., Andersen, A. P., van der Meer, D., \& Bohr, T. (2009). Bubble Pinch-Off in a Rotating Flow. Physical Review Letters, 102(20), 204501. https://doi.org/10.1103/PhysRevLett.102.204501

\section{General rights}

Copyright and moral rights for the publications made accessible in the public portal are retained by the authors and/or other copyright owners and it is a condition of accessing publications that users recognise and abide by the legal requirements associated with these rights.

- Users may download and print one copy of any publication from the public portal for the purpose of private study or research.

- You may not further distribute the material or use it for any profit-making activity or commercial gain

- You may freely distribute the URL identifying the publication in the public portal

If you believe that this document breaches copyright please contact us providing details, and we will remove access to the work immediately and investigate your claim. 


\title{
Bubble Pinch-Off in a Rotating Flow
}

\author{
Raymond Bergmann, ${ }^{1}$ Anders Andersen, ${ }^{1}$ Devaraj van der Meer, ${ }^{2}$ and Tomas Bohr ${ }^{1}$ \\ ${ }^{1}$ Department of Physics and Center for Fluid Dynamics, The Technical University of Denmark, DK-2800 Kgs. Lyngby, Denmark \\ ${ }^{2}$ Physics of Fluids Group and J.M. Burgers Centre for Fluid Dynamics, Department of Science, University of Twente, \\ P.O. Box 217, 7500 AE Enschede, The Netherlands
}

(Received 23 December 2008; revised manuscript received 12 March 2009; published 22 May 2009)

\begin{abstract}
We create air bubbles at the tip of a "bathtub vortex" which reaches to a finite depth. The bathtub vortex is formed by letting water drain through a small hole at the bottom of a rotating cylindrical container. The tip of the needlelike surface dip is unstable at high rotation rates and releases bubbles which are carried down by the flow. Using high-speed imaging we find that the minimal neck radius of the unstable tip decreases in time as a power law with an exponent close to $1 / 3$. This exponent was found by Gordillo et al. [Phys. Rev. Lett. 95, 194501 (2005)] to govern gas flow driven pinch-off, and indeed we find that the volume oscillations of the tip creates a considerable air flow through the neck. We argue that the Bernoulli pressure reduction caused by this air flow can become sufficient to overcome the centrifugal forces and cause the final pinch-off.
\end{abstract}

DOI: 10.1103/PhysRevLett.102.204501

PACS numbers: 47.55.db, 47.32.Ef, 47.55.df

Studies of drop and bubble formation in fluids have a long history [1]. In the last two decades considerable interest has been devoted to studies of the dynamics close to pinch-off in the formation of drops [2] and more recently in the formation of bubbles [3-8]. In recent work Andersen et al. $[9,10]$ reported an interesting bubble forming instability in which the needlelike tip of a so-called "bathtub vortex" becomes unstable and leads to periodic formation of small air bubbles. The system is characterized by high central rotation rate and fast air flow from the tip during the bubble formation. It allows for well controlled measurements of these fundamental effects on pinch-off dynamics. In addition, the system has potential as a bubble generator in which both bubble size and formation frequency may, through the rotation rate, be more easily controlled than in traditional bubble generators like the flow-focusing device $[11,12]$.

In our experiment a cylindrical container with radius $95 \mathrm{~mm}$ is filled with water to a constant level of $240 \mathrm{~mm}$. The container has a small circular hole with radius $R_{\text {drain }}$ of typically $1.2 \mathrm{~mm}$ in the center of the bottom. Water drains under its own weight out of the container at a rate which is approximately $10 \%$ smaller than the prediction using Torricelli's theorem $\left(9 \times 10^{-6} \mathrm{~m}^{3} / \mathrm{s}\right.$ for the $1.2 \mathrm{~mm}$ drain $)$ [10]. We use a recirculation system to maintain a constant water level without disturbing the free surface $[9,10]$. The container is rotated about its vertical symmetry axis with constant rotation rate $\Omega$. After an initial transient an intense vortex with high rotation rate, i.e., typically thousand times larger than $\Omega$, is formed above the drain hole and the free surface is deformed [Fig. 1]. By increasing $\Omega$ the airfilled core penetrates deeper and narrows as it evolves from a stable state (a) to an unstable bubbling state (b) and (c). A further increase of $\Omega$ eventually makes the air-filled core reach all the way to the drain hole, which limits the range in which the bubbling phenomenon can be observed. In the bubbling state near the transition the air-filled neck is stable for considerable time, until a pinch-off is initiated and a bubble is released and carried downward by the draining flow. Due to this downward flow the geometry is similar to the one encountered in the production of bubbles in a co-flow situation [11-13]. In our experiment this effect is not due to external geometry, but comes from the rapid central rotation which creates a funnel-like down flow near the tip, and also, through the centrifugal force which opposes the final pinch-off. As we increase $\Omega$ the tip approaches the drain hole and, similar to the co-flow situation, the increased downward flow will create a more unstable situation where capillary waves are excited [10]. The time evolution of the free surface during a typical

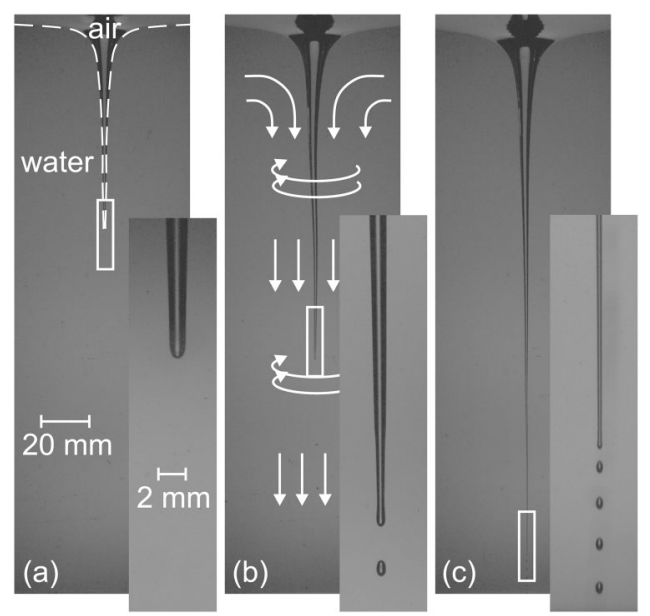

FIG. 1. By increasing the rotation rate while keeping $R_{\text {drain }}=$ $1.2 \mathrm{~mm}$, the air-filled tip of the bathtub vortex goes from a stable state at $13.5 \mathrm{rpm}$ (a) to an unstable bubbling state at $24 \mathrm{rpm}$ (b) and $30.5 \mathrm{rpm}$ (c). In the bubbling state the frequency of bubble formation increases and the bubble size decreases with increasing rotation rate as shown in (b) and (c) for typical cases. 
bubble pinch-off event is shown in detail in Fig. 2. It is the influence of rotation and air flow on the pinch-off dynamics that will be the focus of this Letter.

Figure 3 shows the minimal neck radius, $R$, as function of the time to pinch-off, $\tau$. It is clear from the log-log plot that the minimal neck radius exhibits power-law behavior $R \propto \tau^{\alpha}$ close to the pinch-off for approximately two decades with an exponent $\alpha \approx 1 / 3$ as indicated by the line. Similar behavior is observed over a large range of $\Omega$ from 13.5 to $42 \mathrm{rpm}$ and $R_{\text {drain }}$ ranging from 1.0 to $1.2 \mathrm{~mm}$. Analysis of our data gives a distribution of estimated exponents with mean 0.34 and standard deviation 0.07 (inset of Fig. 3). We believe the spread of exponents is simply noise, and note that the mean value $\alpha=0.34$ is determined to within an uncertainty of only 0.01 .

To understand the observed power law, we start from the Rayleigh-Plesset equation for the collapse of a cylindrical cavity $[14,15]$. This approach is known to yield the correct power laws for the dynamics of the neck radius over a range of different regimes [3]. When inertia and surface tension balance, a power-law exponent $\alpha=2 / 3$ is found, for a balance of viscosity and surface tension, $\alpha=1$ is found, and a purely inertial collapse, gives $\alpha=1 / 2$. The last exponent of $1 / 2$ for the inertial collapse of the bubble is the asymptotic value derived by Eggers et al. [7] and a good approximation of the collapse dynamics proposed by Gordillo et al. [4] and Bergmann et al. [6]. In the following we show how to adapt the Rayleigh-Plesset model to describe the pinch-off of the rotating air-filled neck at the tip of the bathtub vortex.

We use cylindrical polar coordinates $r, \theta, z$ and denote the velocity components $v_{r}, v_{\theta}, v_{z}$. We assume that the flow has rotational symmetry and is independent of height. In addition we assume that the axial flow can be neglected in the last stages of the collapse, and we therefore set $v_{z}=0$. From the continuity equation it then follows that $v_{r}=R \dot{R} / r$, where we let $R$ denote the surface of the cylindrical neck. We can now integrate the radial NavierStokes equation with respect to $r$ from $R$ to $R_{\infty}$ and obtain the Rayleigh-Plesset type equation

$$
\begin{aligned}
& \frac{d(R \dot{R})}{d t} \ln \frac{R}{R_{\infty}}+\frac{1}{2} \dot{R}^{2}\left(1-\frac{R^{2}}{R_{\infty}^{2}}\right)-\frac{2 \eta_{l} \dot{R}+\sigma}{\rho_{l} R} \\
& =-\int_{R}^{R_{\infty}} \frac{v_{\theta}^{2}}{r} d r+\frac{p_{\infty}-p}{\rho_{l}},
\end{aligned}
$$

where $\rho_{l}$ and $\eta_{l}$ denote the density and the dynamic viscosity of the liquid, respectively, $\sigma$ denotes the surface tension, $p_{\infty}$ denotes the pressure in the liquid at the distance $R_{\infty}$, and $p$ denotes the pressure in the air-filled neck. Equation (1) reduces to the well-known cylindrical Rayleigh-Plesset equation in the absence of rotation $[3,15]$. We also note that this equation captures the logarithmic corrections to the power laws when $R_{\infty}$ is identified as a local length scale such that $R / R_{\infty}$ is the local aspect ratio of the cavity [1,7]. To complete the model we must determine $p$ and supplement Eq. (1) with the azimuthal

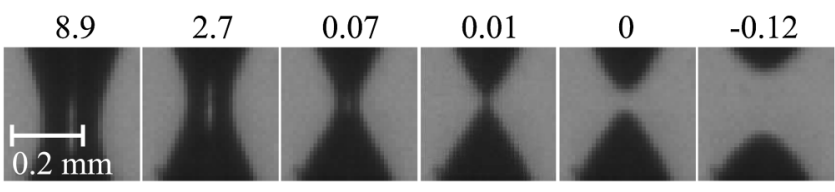

FIG. 2. Bubble pinch-off at the tip of the bathtub vortex for $R_{\text {drain }}=1.2 \mathrm{~mm}$ and $\Omega=13.5 \mathrm{rpm}$. The time to pinch-off in milliseconds is shown above each frame. Note that the axial downflow around the tip does not give rise to any apparent updown asymmetry in the pinch-off region, and that we observe a symmetric pinch-off as expected for bubble formation.

Navier-Stokes equation. To do so, we will first describe our measurements of the stabilizing azimuthal velocity term.

It is impossible to measure the velocity field in our rapidly rotating free surface flow using tracer particles, because the rotation drives heavier particles outwards and forces lighter particles to the center even with a very small mismatch between the particle and the liquid densities. Thereby the lighter particles aggregate at the surface, completely immobilize it, and prevent the collapse. To circumvent this difficulty we generate bubbles with a typical size of $40 \mu \mathrm{m}$ through electrolysis [16]. The rotation slowly drives these bubbles towards the center, where they coalesce with the surface in contrast to the particles which immobilize it. With this method we were able to measure the flow in the bulk as well as at the surface [Fig. 4].

The dash-dot line in Fig. 4(a) shows that the vortex below and on the surface of the tip has a core with solid body rotation (induced by the stagnation point below the tip) of a high central rotation rate of $2 \times 10^{4} \mathrm{rpm}$, i.e., a rotation rate which is thousand times the rotation rate of $19 \mathrm{rpm}$ for the container. The radius of the vortex core is comparable to $R_{\text {drain }}$, and outside this region $v_{\theta}$ tends to the line vortex of the bulk (dashed line). Figure 4(b) shows that the down-flow is localized to a central region with radius equal to 3 times $R_{\text {drain }}$, and that a stagnant region with weaker down-flow exists below the tip.

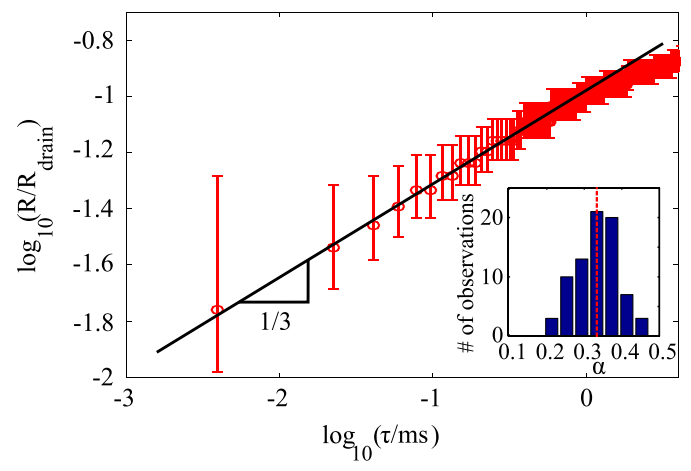

FIG. 3 (color online). Measured radius of the air-filled neck as a function of the time to pinch-off (circles) and power law with exponent $\alpha=1 / 3$ (solid line). $R_{\text {drain }}$ is $1.0 \mathrm{~mm}$ and $\Omega$ is $42 \mathrm{rpm}$. Inset: Frequency histogram for the measured exponents with $\alpha=1 / 3$ indicated. 
The azimuthal Navier-Stokes equation is

$$
\frac{\partial v_{\theta}}{\partial t}+\frac{v_{r}}{r} \frac{\partial\left(r v_{\theta}\right)}{\partial r}=\nu_{l} \frac{\partial}{\partial r}\left(\frac{1}{r} \frac{\partial\left(r v_{\theta}\right)}{\partial r}\right)
$$

where $\nu_{l}$ denotes the kinematic viscosity of the liquid and we have neglected any axial flow. This might seem strange in view of the strong downflow close to the free surface [Fig. 4(b)], but, as can be seen in the movie [17], following a bubble at the collapsing neck, the flow in the pinchregion is shielded from the downflow. The stationary line vortex $v_{\theta}=\Gamma /(2 \pi r)$, with circulation $\Gamma$, is an exact solution expressing angular momentum conservation. One might expect the velocity on the collapsing surface to be of this form, but actually the azimuthal velocity first decreases and then increases when the neck collapses as shown in Fig. 4(c). This is probably due to viscous dissipation induced by the presence of a viscous core outside the narrow, collapsing neck region. To model this in a simplistic way, we approximate the viscous term in Eq. (2) as a finite difference from the center, i.e., as $-\nu_{e} v_{\theta} r^{-2}$ with an effective $\nu_{e}$ of the order of $\nu_{l}$. For a line vortex this would strongly overestimate the dissipation, but in Fig. 4(a) we see that the flow is initially far from a line vortex in the neck region. Using also $v_{r}=R \dot{R} / r$, the resulting equation (written most easily in terms of $r v_{\theta}$ and $r^{2}$ ) can be solved in the region $r>R(t)$ by the method of characteristics as $r v_{\theta}(r, t)=r_{0} v_{\theta}\left(r_{0}, 0\right) \exp \left\{-\nu_{e} \int_{0}^{t} d t^{\prime}\left[R\left(t^{\prime}\right)^{2}+x^{2}\right]^{-1}\right\}$, where $x=\sqrt{R(t)^{2}-r^{2}}$ determines the distance to the free surface. With a power-law collapse $R(t)=R_{0}\left[\left(t_{c}-t\right) / t_{c}\right]^{\alpha}$ and $\alpha \neq 1 / 2$ we obtain the azimuthal velocity on the free surface as

$$
v_{\theta}(R, t)=\frac{\Gamma_{0}}{2 \pi R} \exp \left\{\frac{-t_{c} \nu_{e}}{(1-2 \alpha) R_{0}^{2}}\left[1-\left(\frac{R}{R_{0}}\right)^{\frac{1-2 \alpha}{\alpha}}\right]\right\},
$$

where $\Gamma_{0}=2 \pi R_{0} v_{\theta}\left(R_{0}, 0\right)$. Comparing this result with our flow measurements during the collapse [Fig. 4(c)], with $\alpha=1 / 3$ we find a good agreement when $\nu_{e} \approx 2 \nu_{l}$. Note that the viscous damping initially causes $v_{\theta}$ to de- crease slightly, thereby diminishing the centrifugal pressure and facilitating the collapse. This does not continue indefinitely since $v_{\theta}$ increases rapidly later and near pinchoff tends towards a line vortex as one would have expected from inviscid theory. Thus the rotational term $\int_{R}^{R_{\infty}} v_{\theta}^{2} / r d r$ in Eq. (1) asymptotically scales (at most) as $R^{-2}$ close to pinch-off. As we will argue later, this implies that the contribution of the rotational term is subdominant.

The cause of the collapse is found in the volume oscillations of the tip below the neck. During pinch-off this volume linearly decreases [Fig. 5] and thus gives rise to a constant flux of air through the neck. This creates flow velocities of up to around $1 \mathrm{~m} \mathrm{~s}^{-1}$ with a Bernoulli pressure reduction, $p_{\infty}-p=\rho_{g}\left(v_{g}^{2} / 2+d \phi / d t\right)$, where $\rho_{g}$ is the gas density and $\phi$ is the velocity potential for the gas flow. From the experiment we can estimate this pressure reduction. The first term of the pressure reduction is simply approximated using the average gas-flow velocity $v_{g} \approx Q /\left(\pi R^{2}\right)=-(d V / d t) /\left(\pi R^{2}\right)$, where $V$ is the volume of the tip below the minimal radius. Note that a positive flux $Q$ means an upward flow through the neck. With constant flux $Q$ [Fig. 5], the second term of the pressure reduction depends solely on the dynamics of the neck. The neck can be characterized by $R$ and the radius of curvature $R_{c}$, which from the literature [5-7] and geometrical arguments is expected to approximately scale with $R$. This is confirmed in the experiment, in which the ratio $\delta=R_{c} / R$ is found to be constant ( $\delta \approx 7 \pm 2$ ). This means that the shape of the free surface is preserved to smaller and smaller length scales as the pinch-off is approached, with characteristic neck-length $\sqrt{\delta} R$. For the time-dependent Bernoulli term we therefore find, $d \phi / d t \approx d[\sqrt{\delta} Q /(\pi R)] / d t=-\sqrt{\delta} Q \dot{R} /\left(\pi R^{2}\right)$. We are now ready to determine the behavior of $R(t)$ close to pinch-off. Inserting the expressions for $v_{g}$ and $d \phi / d t$ into Eq. (1), we find terms scaling as $R^{-4}$ and $\dot{R} R^{-2}$, respectively, which means that the rotational $\left(R^{-2}\right)$, viscous $\left(\dot{R} R^{-1}\right)$, and surface tension $\left(R^{-1}\right)$ terms can all be
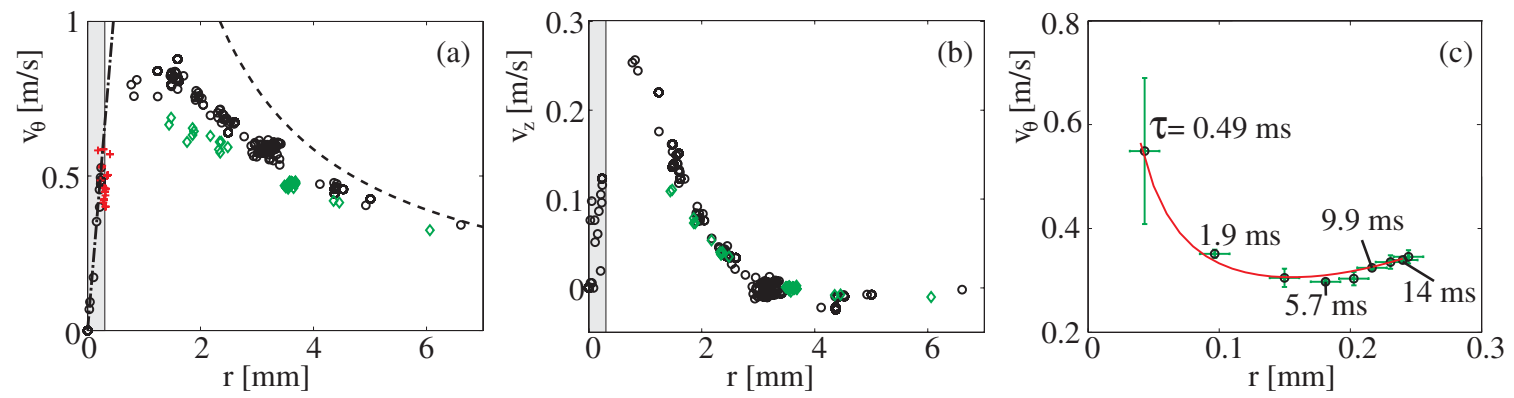

FIG. 4 (color online). Measured azimuthal (a),(c) and axial (b) flow velocities. The measurements in (a) and (b) were made 1 mm above and below the minimal neck radius for $R_{\text {drain }}=1.2 \mathrm{~mm}$ and $\Omega=13.5 \mathrm{rpm}$ (diamonds) and $\Omega=19 \mathrm{rpm}$ (circles, crosses), respectively. The shaded areas indicate the initial radius of the neck before pinch-off. The data within the shaded areas are therefore obtained below the tip, except the red crosses in (a) which are obtained by tracking bubbles on the surface. In (a) we observe solid body rotation around the tip (dash-dot line), while away from the center $v_{\theta}$ tends to the line vortex observed in the bulk (dashed line). Note that in (b) positive $v_{z}$ corresponds to downward flow. (c) shows $v_{\theta}$ of a bubble on the surface of the neck during the collapse ( $\Omega=$ $30.5 \mathrm{rpm}$ ) [17] and agrees with Eq. (3), with $\nu_{e} \approx 1.8 \times 10^{-6} \mathrm{~m}^{2} \mathrm{~s}^{-1}$ (red line). 


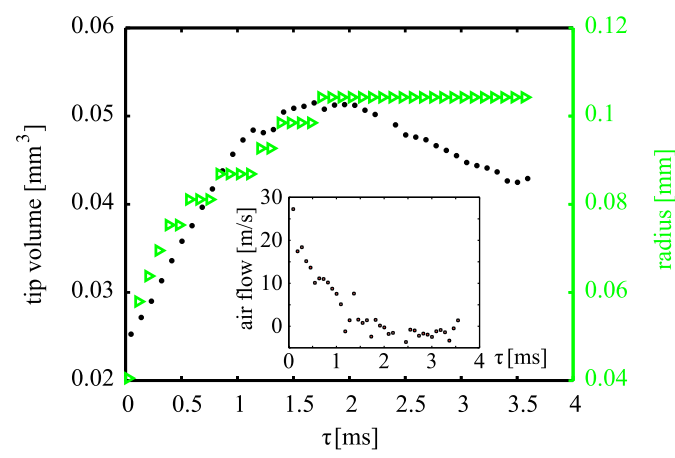

FIG. 5 (color online). Measured minimal neck radius (triangles) and tip volume (filled circles) as functions of time to pinch-off for $R_{\text {drain }}=1.2 \mathrm{~mm}$ and $\Omega=13.5 \mathrm{rpm}$. The compressibility of the air in the tip is negligible, since once the neck reaches the resolution of our recordings $(1 \mu \mathrm{m})$ the air is found to be compressed less than $1 \%$. The average flow velocity through the collapsing neck (inset) is therefore computed directly from the volume change.

neglected, giving

$$
\frac{d(R \dot{R})}{d t} \ln \frac{R}{R_{\infty}}+\frac{1}{2} \dot{R}^{2}\left(1-\frac{R^{2}}{R_{\infty}^{2}}\right) \approx \frac{C_{1}}{R^{4}}-\frac{C_{2} \dot{R}}{R^{2}},
$$

where $C_{1}=Q^{2} \rho_{g} /\left(2 \pi^{2} \rho_{l}\right)$ and $C_{2}=\sqrt{\delta} Q \rho_{g} /\left(\pi \rho_{l}\right)$. To obtain the power-law, including the prefactor, we neglect the $R^{2} / R_{\infty}^{2}$ term and approximate the slowly varying logarithmic term by a constant $\ln \left(R / R_{\infty}\right) \approx-C$. Equation (4) reduces with these assumptions to $-C R \ddot{R}-(C-1 / 2) \dot{R}^{2}=$ $C_{1} / R^{4}-C_{2} \dot{R} / R^{2}$, which has the following power-law solution

$$
\begin{aligned}
R(\tau) & =\left[\frac{3\left(C_{2}+\sqrt{2(1+2 C) C_{1}+C_{2}^{2}}\right)}{1+2 C}\right]^{1 / 3} \tau^{1 / 3} \\
& \approx\left(\frac{3}{\pi \sqrt{1+2 C}}\right)^{1 / 3}|Q|^{1 / 3}\left(\frac{\rho_{g}}{\rho_{l}}\right)^{1 / 6} \tau^{1 / 3},
\end{aligned}
$$

where the approximate equality is valid when $\rho_{g} / \rho_{l}$ is small. The solution indeed has the exponent $\alpha=1 / 3$ in agreement with the experiment. The scaling in Eq. (5) is similar to the result by Gordillo et al. [4] for the gas-flow driven collapse of an asymmetric bubble.

The precise value of $R_{\infty}$ (and therefore $C$ ) is unknown and the low exponent $(1 / 3)$ for $Q$ means that the variations become small. However, comparing to our experiments with a typical gas flow rate $Q=8 \times 10^{-9} \mathrm{~m}^{3} \mathrm{~s}^{-1}$ and taking for simplicity $C=1$ we find that the prefactor is of the right order of magnitude but slightly too small. The prediction agrees well if we use an effective $Q$ that is 2 times (at low $\Omega$ ) to 6 times (at high $\Omega$ ) larger than the measured $Q$. This ties in with the intriguing observation that the pinch-off always occurs while the volume of the tip shrinks $(Q>0)$. In this case the velocity difference between the liquid outside and the gas inside the neck is largest and therefore the effective radius where the upward flow takes place is smallest. This is similar to increasing $Q$.
Also, it is expected, e.g., from the co-flow literature [1113], that this leads to a more unstable interface.

It is interesting to note that the $1 / 3$ power law is actually dictated by dimensional analysis if $Q, \rho_{g}$, and $\rho_{l}$ are the only dimensional control parameters in the problem, i.e., if one would from the outset exclude length scales as $R_{\infty}$ and $R_{0}$ as well as viscous, surface tension and rotational effects. However, the weak $1 / 6$ power-law dependence on the density ratio (first noticed by Gordillo et al. [4]) is surprising and does not follow from dimensional analysis. The low value of this exponent makes it difficult to test this dependence experimentally [18].

In conclusion, we find experimentally that the neck radius close to bubble pinch-off in the bathtub vortex decreases as a power law with an exponent of approximately $1 / 3$. We explain this as a result of the pressure reduction generated by the air flow in the neck, which drives the dynamics and thereby makes the intense rotational flow irrelevant in the final stages of the collapse. We speculate that this is a general and robust feature which is to be expected for bubble pinch-off problems in rotating flows independent of the rotational velocity profile.

R. B. gratefully acknowledges financial support from the Hans Christian Ørsted Postdoc Programme at the Technical University of Denmark.

[1] J. Eggers and E. Villermaux, Rep. Prog. Phys. 71, 036601 (2008).

[2] X. D. Shi, M. P. Brenner, and S. R. Nagel, Science 265, 219 (1994); J. Eggers, Rev. Mod. Phys. 69, 865 (1997).

[3] J. C. Burton, R. Waldrep, and P. Taborek, Phys. Rev. Lett. 94, 184502 (2005).

[4] J. M. Gordillo et al., Phys. Rev. Lett. 95, 194501 (2005).

[5] J. M. Gordillo and M. A. Fontelos, Phys. Rev. Lett. 98, 144503 (2007).

[6] R. Bergmann et al., Phys. Rev. Lett. 96, 154505 (2006).

[7] J. Eggers et al., Phys. Rev. Lett. 98, 094502 (2007).

[8] S. T. Thoroddsen, T. G. Etoh, and K. Takehara, Phys. Fluids 19, 042101 (2007); J. C. Burton and P. Taborek, Phys. Rev. Lett. 101, 214502 (2008).

[9] A. Andersen et al., Phys. Rev. Lett. 91, 104502 (2003).

[10] A. Andersen et al., J. Fluid Mech. 556, 121 (2006).

[11] P. Garstecki et al., Appl. Phys. Lett. 85, 2649 (2004).

[12] B. Dollet et al., Phys. Rev. Lett. 100, 034504 (2008).

[13] A. M. Gañán-Calvo and J. M. Gordillo, Phys. Rev. Lett. 87, 274501 (2001).

[14] Lord Rayleigh, Philos. Mag. 34, 94 (1917).

[15] H. N. Oğuz and A. Prosperetti, J. Fluid Mech. 257, 111 (1993).

[16] W. Merzkirch, Flow Visualization (Academic Press, New York, 1987) 2nd ed.

[17] See EPAPS Document No. E-PRLTAO-102-061922. For more information on EPAPS, see http://www.aip.org/ pubservs/epaps.html.

[18] We repeated our experiment with $\mathrm{SF}_{6}$ gas (density $6.2 \mathrm{~kg} \mathrm{~m}^{-3}$ ) instead of air with no significant change. 